\title{
Randomized controlled study on the curative effects of twist-drill craniotomy and burr-hole craniotomy in the treatment of chronic subdural hematoma
}

\author{
CHANGSONG XU $^{1}$, BING CHEN $^{1}$, LIUJUN XUE ${ }^{1}$, LEI XIA $^{1}$, XIU YANG $^{1}$, \\ MING WEI $^{1}$, XIAOBO HUI ${ }^{2}$, QUAN CHEN ${ }^{1}$, JINLONG ZHENG ${ }^{1}$, ZHENGMING LI $^{2}$, \\ XIANGYANG TIAN ${ }^{1}$, GUANLIANG CHENG ${ }^{1}$, FENG XIAO ${ }^{1}$ and MIN LU $^{3}$ \\ Departments of ${ }^{1}$ Neurology, ${ }^{2}$ Neurosurgery and ${ }^{3}$ Rehabilitation Medicine, \\ Huai'an First People's Hospital, Nanjing Medical University, Huai'an, Jiangsu 223300, P.R. China
}

Received November 4, 2017; Accepted May 2, 2018

DOI: $10.3892 /$ etm.2018.6265

\begin{abstract}
The present randomized controlled study investigated the differences in the curative effects of twist-drill craniotomy (TDC) and burr-hole craniotomy (BHC) in the treatment of chronic subdural hematoma (CSDH). A total of 40 patients diagnosed with CSDH via head computed tomography (CT) who required surgical decompression from January 2016 to January 2017 were enrolled in the present study, and were randomly divided into a TDC group $(n=20)$ and a BHC group $(n=20)$. The modified Rankin scale (mRS) scores of patients were recorded prior to the operation, and at $48 \mathrm{~h}$ and 3 months after the operation. The differences in the $\mathrm{mRS}$ score $\left(\mathrm{V}_{\mathrm{mRS}}\right)$ among the groups were calculated using the Mann-Whitney $\mathrm{U}$ test. The 40 patients enrolled comprised 33 males and 7 females, and there were no significant differences in the general clinical characteristics between the two groups. In the BHC group, 3 patients had a pre-operative mRS score of 5 points, among which 2 cases died at 32 and 45 days after discharge. In the TDC group, 4 patients had a pre-operative mRS score of 5 points, among which 1 case died of epilepsy and pulmonary infection at 1 month after the operation. No difference in the mortality rate was present between the two groups. During the 3-month follow-up, head CT indicated that the intracranial hematoma in a total of 4 patients, including 3 cases in the TDC group and 1 case in the BHC group, completely disappeared. In the BHC group, 3 cases required a repeated incision and drainage after the first operation, while no secondary operation was required in any of the cases of the
\end{abstract}

Correspondence to: Dr Min Lu, Department of Rehabilitation Medicine, Huai'an First People's Hospital, Nanjing Medical University, 6 Beijing Road West, Huai'an, Jiangsu 223300, P.R. China

E-mail: xuchangsong0103@163.com

Key words: chronic subdural hematoma, twist-drill craniotomy, burr-hole craniotomy, minimally invasive, modified Rankin scale score
TDC group. The average length of stay at the hospital (LOS) after TDC was $9.00 \pm 2.91$ days, which was significantly shorter than that after BHC (14.75 \pm 5.95 days). In the total sample of 40 patients, a longer LOS was associated with a higher risk of secondary operation due to recurrence after discharge. The variation value of the mRS score at 3 months after the operation and its ratio vs. the pre-operative score in the TDC group were significantly different from those in the BHC group, suggesting that the improvement of neurological function after TDC was significantly greater than that after BHC. Although 18 patients (90\%) in the TDC group were cured, there was no significant difference from the cure rate in the BHC group [15 patients (75\%)]. In conclusion, no significant differences were identified in the cure rate and the mortality rate of patients with CSDH after the two types of surgical treatment. However, the mRS score in the TDC group at 3 months after the operation exhibited a significantly greater improvement compared with that in the BHC group, and the overall LOS in the TDC group was significantly shorter than that in BHC group. Therefore, TDC is superior to $\mathrm{BHC}$ in the treatment of CSDH (trial registration no. ChiCTR-INR-16008368).

\section{Introduction}

Chronic subdural hematoma (CSDH) occurs frequently in middle-aged and aged people ( $>50$ years), and the incidence rate in individuals aged $>70$ years is 20 times as high as that in the general population (1). Due to the global population aging, a marked increase in the number of CSDH cases is foreseeable (2). Burr-hole craniotomy (BHC) and twist-drill craniotomy (TDC) are two of the most commonly used therapeutic methods for CSDH, and the comparison of their curative effects has always been a hotspot in clinical research.

Evidence-based studies by Weigel et al (3) and Lega et al (4) indicated that BHC is more efficient and safer than TDC. However, an increasing number of studies have suggested that TDC should be used as a preferred clinical regimen. In particular, while the randomized controlled studies of Muzii et al (5), Gökmen et al (6) and Singh et al (7) did not prove that TDC was superior according to the major clinical 
indexes 'recurrence rate' and 'mortality rate'. However, the recent evidence-based studies by Ducruet et al (8) and Almenawer et al (9) pushed the question back to its origin, as the authors argued that TDC has more advantages due to the shorter surgery times and minimally invasive characteristics.

Previous studies generally use non-unified definitions of the outcomes, and among them, the major indexes refer to different clinical implications (3,10-20). For instance, CSDH 'recurrence', the most commonly used index to describe the major outcomes, is often confused with 'secondary operation' $(5,6,18,21)$. To this end, the clinical evaluation system for CSDH was redesigned with the cure rate as the major evaluation index, and the present prospective clinical randomized controlled trial was designed to further determine the advantages and disadvantages of the two therapeutic methods.

\section{Patients and methods}

Definition of TDC. Skull drilling evacuation of hematoma with a skull drilling diameter of $>5 \mathrm{~mm}$ is defined as minimally invasive craniotomy or TDC, that with a drilling diameter of $>5$ and $>30 \mathrm{~mm}$ is defined as BHC and that with larger surgical wounds is defined as general craniotomy. In the flow chart in Fig. 1, the clinical course of patients with $\mathrm{CSDH}$ is illustrated according to previous studies (22).

Definition of recurrent CSDH. The recurrence of CSDH is defined as the repeated accumulation of hematoma in the ipsilateral subdural hematoma cavity confirmed by imaging after the initial treatment. Repeated treatment by decompression drainage in the case of CSDH recurrence with symptoms or aggravated symptoms is known as reoperation. The first operation frequently fails due to certain technical issues or operative complications, including acute subdural hemorrhage, ineffective shunt or poor drainage. At this point, an emergency remedial surgery is required to reconstruct the drainage for intracranial decompression, which is often performed within a short time after the first operation. In the present study, remedial surgery within $48 \mathrm{~h}$ was used as a short-term efficacy index for assessing the success rate of surgery. As a control, the cure rate was used as a long-term index of assessing the surgical efficacy. Patients regarded as cured from CSDH were those with improvement of neurologic impairment after the first operation, including the complete clearance of hematoma without recurrence, residual hematoma in the subdural space identified during follow-up imaging, asymptomatic or no symptom aggravation. Therefore, patients who underwent a second surgery, namely a remedial operation and reoperation, and who died during follow-up, were not deemed as being cured (22).

Inclusion criteria. Patients diagnosed with CSDH via head CT or magnetic resonance imaging (MRI) at Huai'an First People's Hospital (Huai'an China) between January 2016 and January 2017 were enrolled in the present study. Inclusion criteria were a clear correlation of CSDH with neurologic impairment symptoms and signs confirmed via neurological examination; the requirement of hematoma drainage and decompression; and an age $>18$ years for either sex. Patients with CSDH caused by systemic diseases were excluded, and surgical contraindications were excluded through appropriate biochemical examinations, electrocardiogram and chest $\mathrm{CT}$. Patients who met the inclusion criteria were enrolled and randomly grouped after they and their families were informed about the details of the present study, the surgical risks and the relevant safety measures, and provided written informed consent. The present study was approved by the Ethics Committee of Huai'an First People's Hospital.

Random grouping method. A total of 40 random numbers were generated using Statistical Product and Service Solutions (SPSS) v21.0 software (IBM Corp., Armonk, NY, USA). After the patients provided their signature to confirm the experimental scheme, one number was randomly selected from the 40 numbers and the patients were accordingly enrolled into the pre-set groups (23). Three clinicians were independently responsible for the grouping, surgery and follow-up, respectively.

Surgical procedures for TDC. According to the CT scan positioning, the center on the thickest layer was selected as a puncture point and the arteries were avoided. The electric hand drill with a $2-\mathrm{cm}$ minimally invasive intracranial hematoma puncture needle was used to penetrate the skull and dura mater along the locating point to the hematoma center into the subdural hematoma cavity. The drill was then removed, the drainage hose was connected and the drill head was pulled out. The appearance of a dark red bloody fluid overflow was considered to indicate a successful puncture, and the rapid improvement in symptoms were used for verification. The highest point of drainage tube was maintained $10-15 \mathrm{~cm}$ higher than the head puncture point, and the symptoms were improved; the drainage tube was temporarily clipped and then opened after $2 \mathrm{~h}$, and the total drainage time was $48 \mathrm{~h}$. The minimally invasive puncture drainage was successful in 19 patients, and the drainage tube was removed after $48 \mathrm{~h}$ without the traditional irrigation according to previous studies (24-29). Through the routine post-operative review of the head CT, severe complications were excluded and the drainage tube was removed after $48 \mathrm{~h}$.

Surgical procedures for $B H C$. A scalp incision with a length of $\sim 4 \mathrm{~cm}$ was made with a skull drill of $12 \mathrm{~mm}$ in diameter and the drill was slightly expanded by using the rongeur. The dura mater was cut in a cross shape and washed with warm saline, and one drainage tube was placed into it.

Post-operative management. To prevent recurrence, all patients were routinely administered statins every night. After the operation, the tube was clipped for $2 \mathrm{~h}$ after it was opened for drainage for $48 \mathrm{~h}$ and hematoma was significantly reduced on CT; the symptoms were improved and the silicone drainage tube was eventually pulled out. After the operation, in a comfortable supine position, the elderly patients were required to lie in bed and rest, but they were not strictly confined to the bed (11). The incision was disinfected and the dressing was replaced once per day. All 40 patients were followed up by independent clinicians. A new head imaging was reviewed and neurological scoring according to the mRS was performed at 3 months after the operation. 


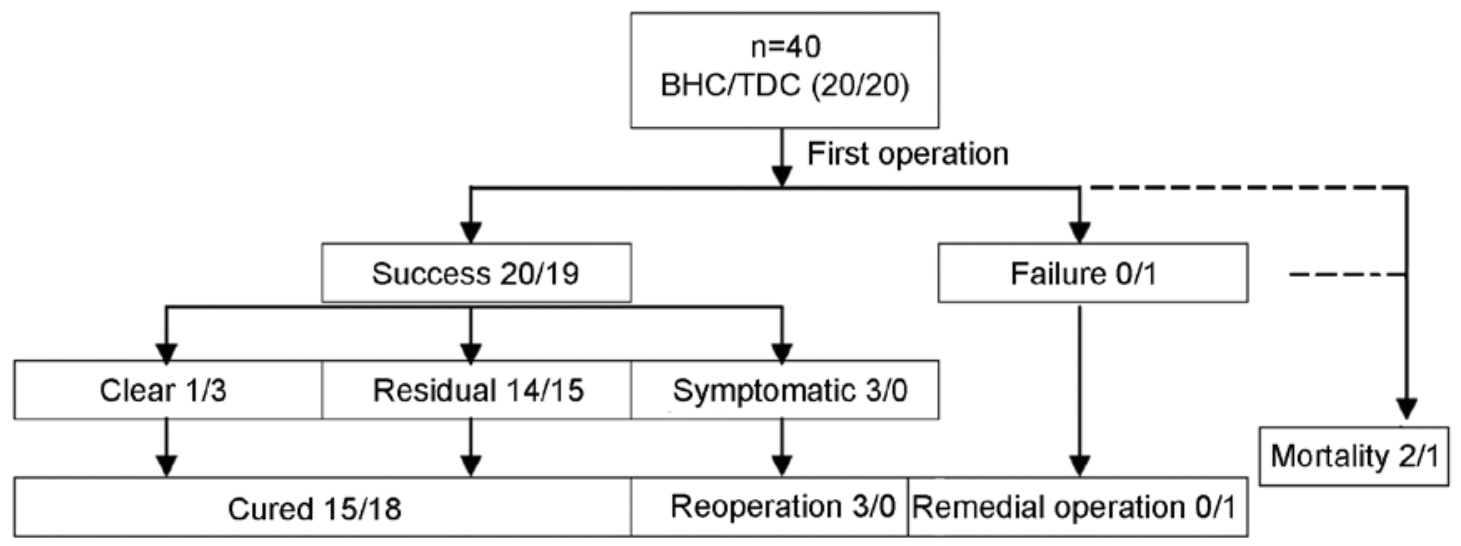

Figure 1. Clinical course of BHC and TDC in the treatment of chronic subdural hematoma. TDC, twist-drill craniotomy; BHC, burr-hole craniotomy.

Statistical analysis. All data from the data set were input into SPSS v21.0 for windows software (IBM Corp.). Measurement data were presented as the mean \pm standard deviation. The independent-samples t-test was used for intergroup comparison and the $\chi^{2}$ test was used for enumeration data. The difference values of mRS scores prior to and after the operation and the ratio vs. the pre-operative value were compared between the two groups using the Mann-Whitney $\mathrm{U}$ test. $\mathrm{P}<0.05$ was considered to indicate a statistically significant difference.

\section{Results}

Demographic data of the patients. A total of 176 cases of CSDH were treated using different surgical methods at Huai'an First People's Hospital from January 2016 to January 2017. This population comprised 132 males and 44 females aged 19-89 years with an average age of $63.2 \pm 10.11$ years. Definite diagnoses were made by brain CT or head MRI examination. On CT, the low-density, equal-density, slightly high-density or mixed-density crescent abnormal fluid areas in the subdural space frequently had a space-occupying effect. The brain parenchyma was pressed, the brain fissure disappeared, and the lateral ventricle and midline structure deviated to the opposite side to varying degrees. The symptoms/complaints of the patients included intracranial hypertension, psychiatric symptoms, hemiplegia and disturbance of consciousness. Among the 40 patients enrolled, 33 were males and 7 were females, and the age ranged from 19-86 years, with 34 cases (85\%) aged $>60$ years and 26 cases $(65 \%)$ with a history of trauma (BHC group, 14 and TDC group, 12). The duration from injury to admission ranged from 3 weeks to 4 months. A total of 14 cases (35\%) denying a history of trauma were aged $>60$ years, and there was a significant age difference between the patients without and with history of trauma $(72.36 \pm 6.37$ vs. $62.73 \pm 15.36$ years; $\mathrm{F}=3.81 ; \mathrm{t}=2.23 ; \mathrm{P}=0.03$ ). It was indicated that a history of trauma may be associated with the formation of CSDH in patients $<60$ years, but not necessarily in patients $>60$ years. A total of $38(95 \%)$ out of 40 patients had unilateral hematoma, while 2 cases (5\%) had bilateral hematoma; no skull fracture and brain parenchymal injury were identified during the imaging examination, and the bleeding volume was 30-210 ml.
Clinical manifestations. A total of 17 cases $(42.5 \%)$ had chronic progressive medical characteristics of intracranial hypertension, including headache, nausea and optic disc edema. A total of 29 cases (72.5\%) presented with focal neurologic impairment due to the hematoma compression, including hemiparesis, epilepsy, alalia and eating difficulty, including 6 cases $(15 \%)$, in which the impairment was severe with manifestations including disturbance of consciousness, psychiatric symptoms, behavioral abnormalities and mental retardation, and 5 cases (12.5\%) with urinary incontinence prior to admission, but whose consciousness as well as respiratory pulse and other vital signs were stable. The medical history of the cases was as follows: 20 cases (50\%) were complicated with varying degrees of hypertension and diabetes mellitus, and 2 cases (5\%) had a history of underlying cerebrovascular disease with unilateral hemiplegia. Furthermore, 2 cases (5\%) had a history of tumor, 1 case (2.5\%) had a history of renal insufficiency and 1 case (2.5\%) used to take antiplatelet drugs, e.g., aspirin, prior to admission. In all patients enrolled in the present study, statin drugs were used to prevent hematoma recurrence. No significant differences in the general clinical characteristics were identified between the two groups (Table I).

Major outcomes. One case in the TDC group received the remedial operation due to no obvious improvement of symptoms and poor hematoma drainage on head CT review at $48 \mathrm{~h}$ after the operation (TDC vs. $\mathrm{BHC} ; \mathrm{P}=0.31$; Table I). However, it cannot be reasoned that the failure rate of TDC was significantly higher than that of BHC. In the BHC group, 3 patients had a pre-operative mRS score of 5 points, among which the hematoma of 2 cases was significantly improved on head CT review at $48 \mathrm{~h}$ after the operation, but the mRS score was still 5 points. Furthermore, 2 patients died at 32 days after discharge (36 days post-operation) and 45 days after discharge (49 days post-operation). In the TDC group, 4 cases had a pre-operative mRS score of 5 points, among which 1 case was discharged 5 days after admission, but his mRS score was 4 points, and he died of epilepsy and pulmonary infection after 1 month. The hemiplegia symptoms of one 80 -year-old case were not alleviated, the patient could not take care of themselves, and the head CT at the 3-month follow-up indicated no increase in the hematoma. No difference in the mortality rate was identified between the 
Table I. Comparison of general clinical characteristics and outcomes between the BHC and TDC groups.

\begin{tabular}{|c|c|c|c|}
\hline Parameter & $\mathrm{BHC}$ & TDC & P-value \\
\hline $\mathrm{n}$ & 20 & 20 & \\
\hline Age (years) & $66.00 \pm 16.74$ & $66.20 \pm 10.11$ & 0.96 \\
\hline Male sex & $17(85)$ & $16(80)$ & 0.68 \\
\hline \multicolumn{4}{|l|}{ Medical characteristics } \\
\hline Trauma & $14(70)$ & $12(60)$ & 0.51 \\
\hline Hypertension & $5(25)$ & $10(50)$ & 0.10 \\
\hline Diabetes & $1(5)$ & 0 & 0.31 \\
\hline Aspirin & 0 & $1(5)$ & 0.31 \\
\hline \multicolumn{4}{|l|}{ mRS score } \\
\hline Pre-operation & $2.55 \pm 1.47$ & $2.74 \pm 1.49$ & 0.70 \\
\hline $48 \mathrm{~h}$ post-operation & $1.35 \pm 1.53$ & $1.42 \pm 1.35$ & 0.88 \\
\hline 3 months post-operation & $1.40 \pm 1.98$ & $0.74 \pm 1.67$ & 0.27 \\
\hline \multicolumn{4}{|l|}{ Outcomes } \\
\hline Remedial surgery & 0 & $1(5)$ & 0.31 \\
\hline Hematoma clear & $1(5)$ & $3(15)$ & 0.29 \\
\hline Hematoma residual & $14(70)$ & $15(75)$ & 0.67 \\
\hline Secondary operation & $3(15)$ & 0 & 0.07 \\
\hline Death & $2(10)$ & $1(5)$ & 0.55 \\
\hline LOS (days) & $14.75 \pm 5.95$ & $9.00 \pm 2.91$ & 0.00 \\
\hline
\end{tabular}

Values are expressed as the mean \pm standard deviation or $\mathrm{n}(\%)$. BHC, burr-hole craniotomy; TDC, twist-drill craniotomy; mRS, modified Rankin scale; LOS, length of stay at hospital.

two groups $(\mathrm{P}=0.55)$. In addition, the head $\mathrm{CT}$ at the 3 -month follow-up indicated that the intracranial hematoma of a total of 4 patients in the two groups was completely removed, including 3 cases in the TDC group and 1 male case aged 19 years in the BHC group (TDC vs. BHC; $\mathrm{P}=0.29$; Table I). In the remaining patients, residual hematoma was present after 3 months (Fig. 2). The BHC group contained 3 cases of intracranial hypertension, 2 of which were diagnosed with a recurrence of $\mathrm{CSDH}$, and received the secondary operation at 30 and 47 days after the first operation. One other case was diagnosed with a secondary intracranial infection and received the incision and drainage again due to fever, scalp incision swelling and other central nervous system infection symptoms, as well as a mixed-density shadow in the subdural space on head CT. In the TDC group, those patients with improved symptoms within $48 \mathrm{~h}$ of surgery had stable symptoms until the 3-month follow-up, and none of the patients was required to undergo a secondary operation (TDC vs. BHC; $\mathrm{P}=0.07$ ). It was also identified that patients with a high pre-operative score retained these increased scores at $48 \mathrm{~h}$ after the operation $(\mathrm{P}<0.001)$, indicating that the symptoms may be obviously improved by the two types of surgery within $48 \mathrm{~h}$. The average LOS after TDC was $9.00 \pm 2.91$ days, which was significantly shorter than that after BHC $(14.75 \pm 5.95$ days; $\mathrm{P}<0.01)$. To further compare the curative effects of the two types of operation, the differences in $\mathrm{mRS}$ scores prior to the operation vs. $48 \mathrm{~h}$ post-operation $\left(\mathrm{V}_{\text {pre-48 }}\right)$, prior to the operation vs. 3 months post-operation $\left(\mathrm{V}_{\text {pre-3 m }}\right)$ and $48 \mathrm{~h}$ vs. 3 months after the operation $\left(\mathrm{V}_{48 \mathrm{~h}-3 \mathrm{~m}}\right)$ were determined, and the ratio of these three variables vs. the pre-operative mRS score was also calculated $\left[\mathrm{V}_{(\text {pre- } 48 \mathrm{~h}) / \mathrm{pre}}\right.$, $\mathrm{V}_{\text {(pre- } 3 \mathrm{~m}) / \text { pre }}$ and $\mathrm{V}_{(48 \mathrm{~h}-3 \mathrm{~m}) / \text { pre }}$, respectively]. The Mann-Whitney $\mathrm{U}$ test was performed and the results indicated that the variation values $\mathrm{V}_{\text {pre-3 } \mathrm{m}}$ and $\mathrm{V}_{48 \mathrm{~h}-3 \mathrm{~m}}$ of the mRS sore at 3 months after the operation and the ratios $\mathrm{V}_{(\text {pre- } 3 \mathrm{~m}) / \mathrm{pre}}$ and $\mathrm{V}_{(48 \mathrm{~h}-3 \mathrm{~m}) / \mathrm{pre}}$ in the TDC group were obviously different compared with those in the BHC group $(\mathrm{P}<0.05$; Table II), suggesting that the improvement of neurological function in the TDC group after the operation was more obvious than that in the BHC group. According to the traditional definition, the remedial operation (1 case in the TDC group) and secondary operation (3 cases in the BHC group) were considered to indicate a recurrence of $\mathrm{CSDH}$ in the present study, and the recurrence rate was not significantly different between the two groups $(\mathrm{P}=0.29$; Table I). Although 18 patients $(90 \%)$ in the TDC group were cured (the operation was successful and the survival time was $>3$ months without recurrence), there was no significant difference in the cure rate compared with that in the BHC group $(\mathrm{P}=0.21)$.

\section{Discussion}

CSDH is a common neurological disease and frequently occurs in the elderly. With the global population aging, its incidence rate is high. BHC and TDC are the two most commonly used drainage techniques for subdural hematoma. Due to non-unified definitions and inconsistent evaluation indexes, previous studies as a whole have not convincingly demonstrated which 
Table II. Comparison of $\mathrm{V}_{\mathrm{mRS}}$ between the two surgery groups.

\begin{tabular}{|c|c|c|c|c|c|c|c|}
\hline \multirow[b]{2}{*}{$\mathrm{V}_{\mathrm{mRS}}$} & \multicolumn{3}{|c|}{$\mathrm{BHC}$} & \multicolumn{3}{|c|}{ TDC } & \multirow[b]{2}{*}{ P-value } \\
\hline & $\mathrm{n}$ & Mean & Sum & $\mathrm{n}$ & Mean & Sum & \\
\hline $\mathrm{V}_{\text {pre-48 h }}$ & 20 & 19.93 & 398.00 & 20 & 21.08 & 421.00 & 0.72 \\
\hline $\mathrm{V}_{\text {pre-3 m }}$ & 20 & 16.40 & 328.00 & 19 & 23.79 & 452.00 & 0.04 \\
\hline $\mathrm{V}_{48 \mathrm{~h}-3 \mathrm{~m}}$ & 20 & 16.23 & 32450 & 19 & 23.97 & 455.50 & 0.03 \\
\hline $\mathrm{V}_{(\text {pre-48 h)/pre }}$ & 20 & 22.03 & 440.50 & 20 & 18.98 & 379.50 & 0.40 \\
\hline $\mathrm{V}_{(\text {pre-3 } \mathrm{m}) / \mathrm{pre}}$ & 20 & 16.68 & 333.50 & 19 & 23.50 & 446.50 & 0.03 \\
\hline $\mathrm{V}_{(48 \mathrm{~h}-3 \mathrm{~m}) / \mathrm{pre}}$ & 20 & 15.70 & 314.00 & 19 & 24.53 & 466.00 & 0.01 \\
\hline
\end{tabular}

The Mann-Whitney U test was applied. BHC, burr-hole craniotomy; TDC, twist-drill craniotomy; mRS, modified Rankin scale; $\mathrm{V}_{\mathrm{mRS}}$, change in $\mathrm{mRS}$ score; pre, prior to surgery; $3 \mathrm{~m}, 3$ months after treatment.
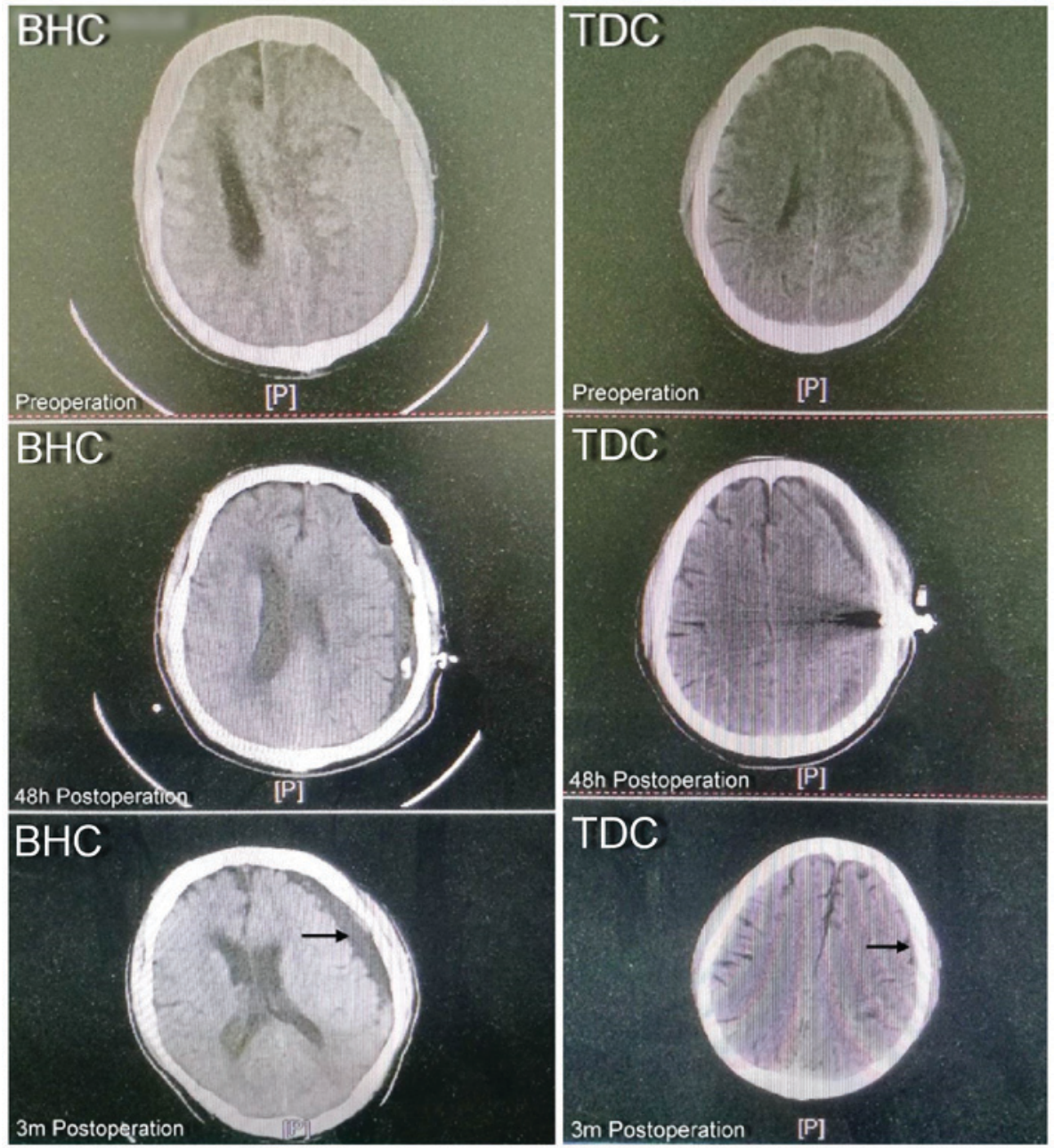

Figure 2. Computed tomography images prior to the operation and at $48 \mathrm{~h}$ and 3 months after BHC and TDC. Residual hematomas were observed in the BHC group. TDC, twist-drill craniotomy; BHC, burr-hole craniotomy; [P], posterior.

one of the two techniques provides more benefits (22). Three randomized controlled studies had the following shortcomings: The definition of recurrence in the study by Muzii et al (5) was based on the imaging results, which is different from the traditional evaluation based on symptom recurrence; however, the other indexes in their study were evaluated in a similar manner. In the study by Gökmen et al (6), the recurrence of CSDH was considered as requirement for a secondary operation. The study by Singh et al (7) had similar clinical implications to Gökmen et al (6), but the detailed description of definitions was lacking. Oh et al (14) identified in a review that as an inconsistency, the definition of 'recurrence' mainly included remedial operation, remnants and secondary operation. Although the recurrence rate has been a major index for 
evaluating the efficacy in the past, it has also been the most inconsistently defined parameter.

The definition of recurrence based on residual hematoma may be the major cause for the variety of results. In the present study, the proportion of patients with clear intracranial residual hematoma on head $\mathrm{CT}$ at the 3-month follow-up after the operation was $72.5 \%$, which was consistent with the results of Weigel et al $(3,20)$. In previous studies, the presence of post-operative residual hematoma is the most common clinical outcome for patients receiving treatment for CSDH. At present, it remains to be clarified whether the residual hematoma is the unabsorbed hematoma after the first onset and treatment, compensatory product formed due to the difficult re-expansion and failed filling of residual cavity in patients with brain atrophy or new subdural hematoma. Since the recurrence of CSDH was determined based on residual hematoma in the past (11,24-29) and monitoring of the changes in residual hematoma varies, the definition of 'recurrence' is not consistent among studies.

In order to avoid confusing results due to the unclear definition of recurrence, the terminology of the present study was based on studies by Neal et al (30) and Singla et al (31), and a novel index system was established. As one of the major indexes for evaluating the long-term efficacy of surgery, patients with removal of $\mathrm{CSDH}$ or residual $\mathrm{CSDH}$ without any new neurologic impairment in the follow-up imaging after 3 months were deemed as cured. The cured patients only underwent one operation. No additional surgery was required based on the examination outcomes, including various criteria for assessing the recurrence and reasons for determining a secondary operation. The cure rate was used as the major index for evaluating the efficacy, which has applicability in the clinic.

Based on the above, the results of the present study indicated that 75 and $90 \%$ of patients in the BHC group and TDC group, respectively, were cured, and there was no statistically significant difference between the two groups. However, it is indicated that BHC and TDC do not have the same effectiveness. Therefore, the improvement level of the mRS score, expressed as the $\mathrm{V}_{\mathrm{mRS}}$ value, was used as another evaluation index with the aim to clarify the effectiveness of the two surgeries through further evaluating the overall changes in $\mathrm{mRS}$ scores prior to and after the two types of operation in each group.

The differences in mRS scores between the time-points prior to the operation, at $48 \mathrm{~h}$ and 3 months after operation were calculated $\left(\mathrm{V}_{\text {pre-48 }}, \mathrm{V}_{\text {pre-3 } \mathrm{m}}\right.$ and $\left.\mathrm{V}_{48 \mathrm{~h}-3 \mathrm{~m}}\right)$, and the ratio of these three variables to the pre-operative mRS score was also calculated $\left[\mathrm{V}_{(\text {pre- } 48 \mathrm{~h}) / \mathrm{pre}}, \mathrm{V}_{(\text {pre- } 3 \mathrm{~m}) / \text { pre }}\right.$ and $\left.\mathrm{V}_{(48 \mathrm{~h}-3 \mathrm{~m}) / \mathrm{pre}}\right]$. The Mann-Whitney U test was performed and the results indicated that the variation values $\mathrm{V}_{\text {pre-3 } \mathrm{m}}$ and $\mathrm{V}_{48 \mathrm{~h}-3 \mathrm{~m}}$ of $\mathrm{mRS}$ score at 3 months after the operation and the ratios $V_{(\text {pre-3 m)/pre }}$ and $\mathrm{V}_{(48 \mathrm{~h}-3 \mathrm{~m}) / \mathrm{pre}}$ in the TDC group exhibited obvious differences compared with those in the BHC group $(\mathrm{P}<0.05)$, suggesting that the improvement of neurological function in the TDC group at 3 months after the operation was more obvious than that in the BHC group. Therefore, it may be assumed that with the increase of sample size, the long-term cure rate in the TDC group may be superior to that in BHC group. This present result is superior to that of the recent study by Wang et al (32), which indicated that TDC and BHC have comparable clinical outcomes in the treatment of patients with CSDH.
As previous studies, the present study identified no significant difference in the mortality rate of $\mathrm{CSDH}$ patients after treatment with the two different surgeries $(3,8)$. In the present study, the overall failure rate of TDC was 5\%, which was within the previously reported range (0-36.4\%) (8). The present study also proved that the success rate of the first BHC was higher (100\%), but it did not suggest that the failure rate of TDC is higher than that of $\mathrm{BHC}(\mathrm{P}=0.31)$. The failure rate may be associated with factors including the surgical conditions, proficiency of the surgeon, technical factors and the overall condition of the patients.

In the present study, the rate of symptom recurrence and secondary operation within 3 months after BHC was up to $15 \%$, which was consistent with the fact that the long-term improvement in mRS score after the operation in the BHC group was lower than that in the TDC group, proving the rationality and completeness of the evaluation system applied. However, it may not be reasonable to use the secondary operation rate as a major clinical index to assess the efficacy of the first operation. In addition, as mentioned above, among previous studies, the definition of 'secondary operation' is as inconsistent as that of 'recurrence', and it lacks the practicality for clinical use as an index for measuring the surgical efficacy.

In the present study, no significant differences were identified in the cure rate and the mortality rate of patients with CSDH after the two types of surgical treatment. However, the mRS score in the TDC group at 3 months after the operation was significantly more improved compared with that in the BHC group, and the overall LOS in the TDC group was significantly shorter compared with that in the BHC group. Therefore, the $\mathrm{TDC}$ regimen was indicated to be superior to the $\mathrm{BHC}$ regimen.

In conclusion, the effectiveness of TDC in the treatment of $\mathrm{CSDH}$ is not lower than that of $\mathrm{BHC}$, and it is characterized by a simpler operation and smaller damage; at the same time, the LOS of patients receiving TDC is also obviously shorter than that of patients after BHC, and the improvement of neurological score of patients who received TDC was better in the long-term. Therefore, TDC is superior to $\mathrm{BHC}$ for the treatment of CSDH.

\section{Acknowledgements}

Not applicable.

\section{Funding}

The present study was supported by the Key Project of Science and Technology Development Fund of Nanjing Medical University (grant no. 2015NJMUZD074).

\section{Availability of data and materials}

All data generated or analyzed during this study are included in this published article.

\section{Authors' contributions}

CX, BC, LXu, LXi and XY were involved in the study design; MW, XH, QC, JZ, ML and ZL were involved in data collection; XT, GC and FX were involved in data analysis; CX and 
ML prepared the manuscript. All authors read and approved the final manuscript.

\section{Ethics approval and consent to participate}

This study was approved by the Ethics Committee of Huai'an First People's Hospital. Written informed consent was obtained from the patients and/or their guardians.

\section{Patient consent for publication}

Patients or their guardians provided written informed consent for publication.

\section{Competing interests}

The authors declare that they have no competing interests.

\section{References}

1. Abecassis IJ and Kim LJ: Craniotomy for treatment of chronic subdural hematoma. Neurosurg Clin N Am 28: 229-237, 2017.

2. Santarius T and Hutchinson PJ: Chronic subdural haematoma: Time to rationalize treatment? Br J Neurosurg 18: 328-332, 2004

3 . Weigel R, Schmiedek P and Krauss JK: Outcome of contemporary surgery for chronic subdural haematoma: Evidence based review. J Neurol Neurosurg Psychiatry 74: 937-943, 2003.

4. Lega BC, Danish SF, Malhotra NR, Sonnad SS and Stein SC: Choosing the best operation for chronic subdural hematoma: A decision analysis. J Neurosurg 113: 615-621, 2010.

5. Muzii VF, Bistazzoni S, Zalaffi A, Carangelo B, Mariottini A and Palma L: Chronic subdural hematoma: Comparison of two surgical techniques. Preliminary results of a prospective randomized study. J Neurosurg Sci 49: 41-47, 2005.

6. Gökmen M, Sucu HK, Ergin A, Gokmen A and Bezircio Lu H: Randomized comparative study of burr-hole craniostomy versus twist drill craniostomy; surgical management of unilateral hemispheric chronic subdural hematomas. Zentralbl Neurochir 69: 129-133, 2008.

7. Singh SK, Sinha M, Singh VK, Parihar A, Srivastava C, Ojha BK and Chandra A: A randomized study of twist drill versus burr hole craniostomy for treatment of chronic subdural hematomas in 100 patients. Indian J Neurotrauma 8: 83-88, 2011.

8. Ducruet AF, Grobelny BT, Zacharia BE, Hickman ZL, DeRosa PL, Andersen KN, Sussman E, Carpenter A and Connolly EJ Jr: The surgical management of chronic subdural hematoma. Neurosurg Rev 35: 155-169, 2012.

9. Almenawer SA, Farrokhyar F, Hong C, Alhazzani W, Manoranjan B, Yarascavitch B, Arjmand P, Baronia B, Reddy K, Murty N and Singh S: Chronic subdural hematoma management: A systematic review and meta-analysis of 34,829 patients. Ann Surg 259: 449-457, 2014.

10. Xu CS: Inconsistent data resources weaken the quality of research results. Ann Surg 262: e121-e122, 2015.

11. Abouzari M, Rashidi A, Rezaii J, Esfandiari K, Asadollahi M, Aleali $\mathrm{H}$ and Abdollahzadeh $\mathrm{M}$ : The role of postoperative patient posture in the recurrence of traumatic chronic subdural hematoma after burr-hole surgery. Neurosurgery 61: 794-797, 2007.

12. Lin X: Comparing twist-drill drainage with burr hole drainage for chronic subdural hematoma. Chin J Traumatol 14: 170-173, 2011.

13. Nakajima H, Yasui T, Nishikawa M, Kishi $H$ and Kan M: The role of postoperative patient posture in the recurrence of chronic subdural hematoma: A prospective randomized trial. Surg Neurol 58: 385-387, 387, 2002
14. Oh HJ, Lee KS, Shim JJ, Yoon SM, Yun IG and Bae HG: Postoperative course and recurrence of chronic subdural hematoma. J Korean Neurosurg Soc 48: 518-523, 2010.

15. Oishi M, Toyama M, Tamatani S, Kitazawa T and Saito M: Clinical factors of recurrent chronic subdural hematoma. Neurol Med Chir (Tokyo) 41: 382-386, 2001.

16. Ramachandran $\mathrm{R}$ and Hegde T: Chronic subdural hematomas-causes of morbidity and mortality. Surg Neurol 67: 367-373, 2007.

17. Rohde V, Graf G and Hassler W: Complications of burr-hole craniostomy and closed-system drainage for chronic subdural hematomas: A retrospective analysis of 376 patients. Neurosurg Rev 25: 89-94, 2002

18. Santarius T, Kirkpatrick PJ, Ganesan D, Chia HL, Jalloh I, Smielewski P, Richards HK, Marcus H, Parker RA, Price SJ, et al: Use of drains versus no drains after burr-hole evacuation of chronic subdural haematoma: A randomised controlled trial. Lancet 374: 1067-1073, 2009.

19. Torihashi K, Sadamasa N, Yoshida K, Narumi O, Chin M and Yamagata S: Independent predictors for recurrence of chronic subdural hematoma: A review of 343 consecutive surgical cases. Neurosurgery 63: 1125-1129, 2008.

20. Weigel R, Krauss JK and Schmiedek P: Concepts of neurosurgical management of chronic subdural haematoma: Historical perspectives. Br J Neurosurg 18: 8-18, 2004.

21. Rughani AI, Lin C, Dumont TM, Penar PL, Horgan MA and Tranmer BI: A case-comparison study of the subdural evacuating port system in treating chronic subdural hematomas. J Neurosurg 113: 609-614, 2010.

22. Xu CS, Lu M, Liu LY, Yao MY, Cheng GL, Tian XY, Xiao F, Wan Q and Chen F: Chronic subdural hematoma management: Clarifying the definitions of outcome measures to better understand treatment efficacy-a systematic review and meta-analysis. Eur Rev Med Pharmacol Sci 21: 809-818, 2017.

23. Jadad AR and Enkin MW: Randomized controlled trials: Questions, answers, and musings, Second Edition. 2008.

24. Erol FS, Topsakal C, Faik OM, Kaplan M and Tiftikci MT: Irrigation vs. closed drainage in the treatment of chronic subdural hematoma. J Clin Neurosci 12: 261-263, 2005.

25. Kuwabara M, Sadatomo T, Yuki K, Migita K, Imada Y, Shimizu K, Hara T, Oba H and Kurisu K: The effect of irrigation solutions on recurrence of chronic subdural hematoma: A consecutive cohort study of 234 patients. Neurol Med Chir (Tokyo) 57: 210-216, 2017

26. Iftikhar M, Siddiqui UT, Rauf MY, Malik AO and Javed G: Comparison of Irrigation versus No Irrigation during burr hole evacuation of chronic subdural hematoma. J Neurol Surg A Cent Eur Neurosurg 77: 416-421, 2016.

27. Kim DH, Kim HS, Choi HJ, Han IH, Cho WH and Nam KH: Recurrence of the chronic subdural hematoma after burr-hole drainage with or without intraoperative saline irrigation. Korean J Neurotrauma 10: 101-105, 2014.

28. Takeda N, Sasaki K, Oikawa A, Aoki N and Hori T: A new simple therapeutic method for chronic subdural hematoma without irrigation and drainage. Acta Neurochir (Wien) 148: 541-546, 2006.

29. Suzuki K, Sugita K, Akai T, Takahata T, Sonobe M and Takahashi S: Treatment of chronic subdural hematoma by closed-system drainage without irrigation. Surg Neurol 50: 231-234, 1998

30. Neal MT, Hsu W, Urban JE, Angelo NM, Sweasey TA and Branch CJ Jr: The subdural evacuation port system: Outcomes from a single institution experience and predictors of success. Clin Neurol Neurosurg 115: 658-664, 2013.

31. Singla A, Jacobsen WP, Yusupov IR and Carter DA: Subdural evacuating port system (SEPS)-minimally invasive approach to the management of chronic/subacute subdural hematomas. Clin Neurol Neurosurg 115: 425-431, 2013.

32. Wang K, Chen D, Cao X and Gao L: A prospective comparative study of twist drill craniostomy versus burr hole craniostomy in patients with chronic subdural hematoma. Turk Neurosurg 27: 60-65, 2017. 